\title{
Technical refinement of prosthetic vascular graft anastomosis to recipient inferior vena cava for secure middle hepatic vein reconstruction in living donor liver transplantation
}

\author{
I-Ji Jeong, Shin Hwang, Tae-Yong Ha, Gi-Won Song, Dong-Hwan Jung, Gil-Chun Park, \\ Chul-Soo Ahn, Deok-Bog Moon, Ki-Hun Kim, Young-In Yoon, and Sung-Gyu Lee
}

\begin{abstract}
Division of Hepatobiliary Surgery and Liver Transplantation, Department of Surgery, Asan Medical Center, University of Ulsan College of Medicine, Seoul, Korea
\end{abstract}

\begin{abstract}
Backgrounds/Aims: Hemashield vascular grafts has been used for middle hepatic vein (MHV) reconstruction during living donor liver transplantation (LDLT). We occasionally encounter outflow disturbance of MHV conduit at the anastomotic stump of the middle-left hepatic vein (MLHV) trunk. To mitigate the disturbance, we carried out a series of studies regarding hemodynamics-compliant MHV reconstruction. Methods: This study comprised of three parts: Part 1: Determining the causes of outflow disturbance; Part 2: Computational simulative analysis; and, Part 3: Clinical application of our refined technique. The types of Hemashield conduit-MLHV stump reconstruction were end-to-end anastomosis (type 1), side-toend anastomosis (type 2), and oblique cutting of the conduit end and patch plasty (type 3). Results: In Part 1 study, the reconstruction types were type 1 in 23 , type 2 in 25, and type 3 in 2 . Significant anastomotic stenosis was identified in $7(30.4 \%)$ in type $1,6(24.0 \%)$ in type 2 , and none $(0 \%)$ in type 3 . The size of MLHV stump was the most important factor for anastomotic stenosis. Through Part 2 study, technical knacks were developed as follows: the conduit end was cut in a dumb-bell shape and a vessel patch attached; and then sutured bidirectionally from the 9 o'clock direction. In Part 3 study, these knacks were applied to 5 patients and none of them experienced noticeable anastomotic stenosis. Conclusions: Our refined technique to perform conduit-MLHV stump anastomosis appears to reduce the risk of anastomotic outflow disturbance for relatively small MLHV stump. (Ann Hepatobiliary Pancreat Surg 2020;24:144-149)
\end{abstract}

Key Words: Prosthetic vascular graft; Middle hepatic vein; Anastomosis; Hepatic venous congestion; Living donor liver transplantation

\section{INTRODUCTION}

Middle hepatic vein (MHV) reconstruction with vascular graft interposition is regarded as one of the standard procedures for living donor liver transplantation (LDLT) using a modified right lobe graft. Various interposition conduit materials, such as autologous and homologous vessels and prosthetic vascular grafts, have been used. ${ }^{1-5}$ Since the supply of homologous vessel allografts is very limited worldwide, various prosthetic vascular grafts are being used instead of homograft and their short-term patency rates were reported to be acceptably high. ${ }^{3-5}$

Despite the diameter of prosthetic vascular grafts being large enough, outflow disturbance of the MHV conduit still occurs unexpectedly. Based on our experience, the usual sites of stenosis were the anastomotic sites between the prosthetic graft and MHV tributary orifices at the right liver graft. However, the anastomotic site between the prosthetic graft and the stump of the middle-left hepatic vein (MLHV) trunk attached at the recipient inferior vena cava (IVC) appeared to be another source of outflow disturbance. Anastomosis of a prosthetic vascular conduit to the right liver graft is performed at the back table. Considering that anastomosis at back table is performed under good work field, the anastomotic stenosis is often associated with small size of the graft MHV tributaries. In contrast, a prosthetic vascular graft is anastomosed with the MLHV stump, which is much larger than the graft

Received: March 9, 2020; Revised: March 13, 2020; Accepted: March 15, 2020

Corresponding author: Shin Hwang

Department of Surgery, Asan Medical Center, University of Ulsan College of Medicine, 88 Olympic-ro 43-gil, Songpa-gu, Seoul 05505, Korea Tel: +82-2-3010-3930, Fax: +82-2-3010-6701, E-mail: shwang@amc.seoul.kr

Copyright (C) 2020 by The Korean Association of Hepato-Biliary-Pancreatic Surgery

This is an Open Access article distributed under the terms of the Creative Commons Attribution Non-Commercial License (http://creativecommons.org/ censes/by-nc/4.0) which permits unrestricted non-commercial use, distribution, and reproduction in any medium, provided the original work is properly cited. Annals of Hepato-Biliary-Pancreatic Surgery - pISSN: 2508-5778 - elSSN: 2508-5859 
MHV tributaries. Due to such large-sized anastomosis, marked outflow disturbance inducing hepatic venous congestion rarely develop. However, the morphological configuration of the conduit-MLHV stump anastomosis is often distorted primarily due to highly variable matching of the prosthetic vascular graft and MLHV stump. Such hemodynamics-non-compliant configuration can induce unwanted raise of outflow resistance, which will be a potential source of MHV outflow disturbance.

To solve such a hemodynamics-associated problem regarding the conduit anastomosis to the MLHV stump, we carried out a series of studies on hemodynamics-compliant reconstruction and established refined techniques enabling secure MHV reconstruction.

\section{MATERIALS AND METHODS}

\section{Study design}

This study comprised of three parts as follows: Part 1:
Determining the underlying causes of outflow disturbance at the conduit anastomotic site of the MLHV stump. Part 2: A hemodynamics-compliant hemodynamics analysis to solve the abovementioned outflow disturbance. Part 3: Clinical application of the refined techniques of MHV reconstruction. This study protocol was approved by the Institutional Review Board of our institution.

\section{Mechanism of MHV anastomotic stenosis (Part 1)}

We selected 50 case of adult LDLT using MHV reconstruction using a collagen-impregnated woven double velour polyester graft (Hemashield Platinum, Maquet; henceforth simply referred to as Hemashield graft) during a 6month study period from July 2018 to December 2018. The morphological configuration of the conduit anastomotic site was analyzed using serial follow-up of dynamic liver computed tomography (CT) scans (Figs. 1, 2).
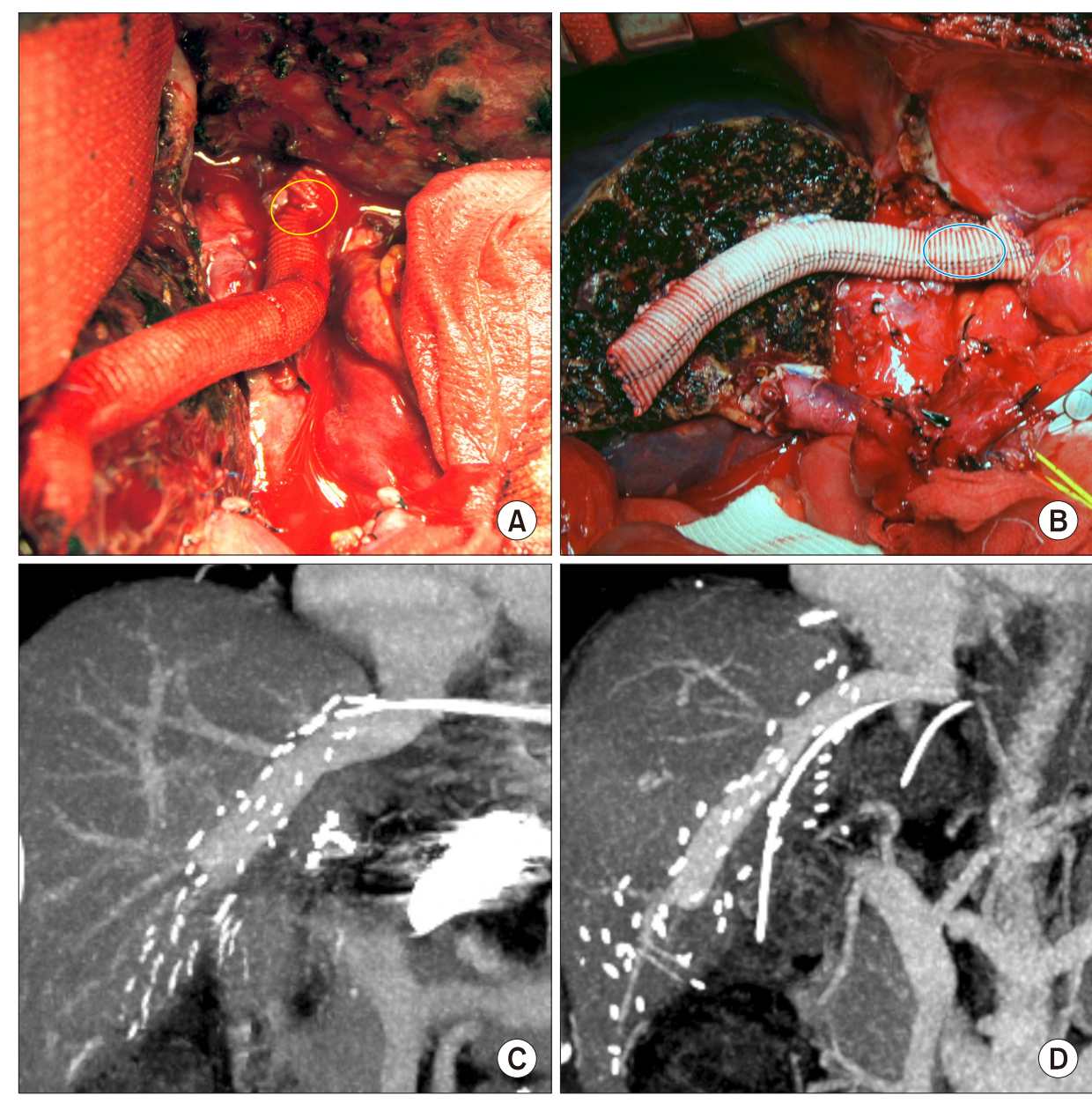

Fig. 1. Intraoperative photographs and computed tomography images taken 1 week after liver transplantation. The reconstruction techniques were type 1 (A and C) and type 2 (B and D). Dotted circles indicate the outlines of the anastomosis. 

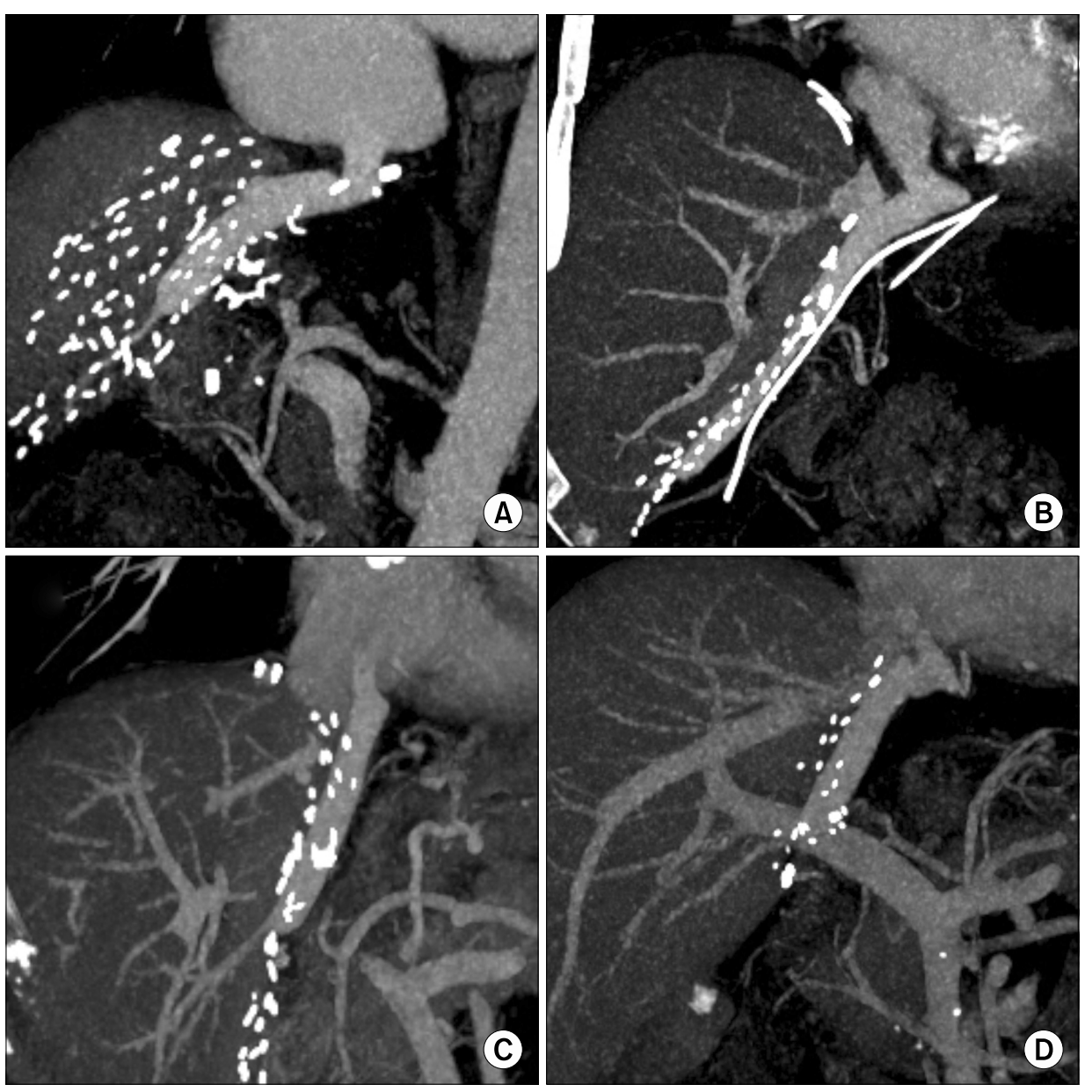

Fig. 2. Comparison of dynamic computed tomography images taken 2-3 weeks after liver transplantation. In type 1 reconstruction technique, the anastomosis is wide (A) and narrow (C). In type 2 reconstruction technique, the anastomosis is wide (B) and narrow (D).

Type 1

Type 2

Type 3
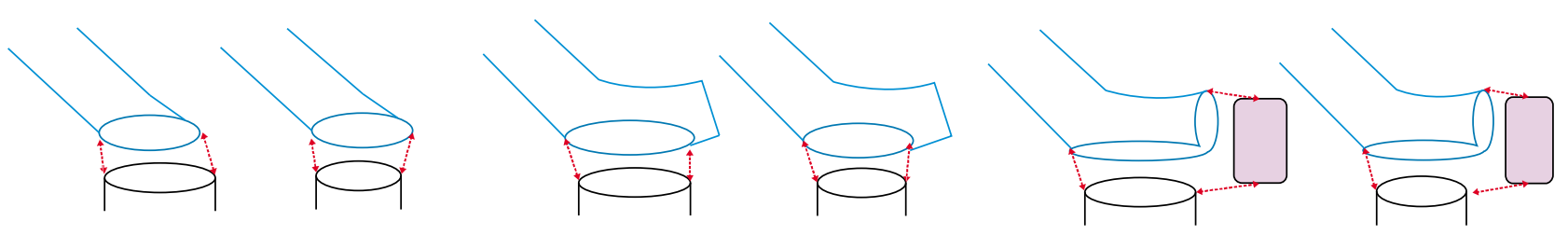

Fig. 3. Schematic illustration of the reconstruction techniques for anastomosis of a Hemashield conduit and the left-middle hepatic vein trunk stump. A colored rectangular patch indicates a vein homograft.

\section{Computational simulative analysis (Part 2)}

There were three types of conduit-MLHV stump reconstruction techniques in the Part 1 study: the first was end-to-end anastomosis with oblique cutting of the conduit end (type 1). The second was side-to-end anastomosis (type 2), and the third was oblique cutting of the conduit end and add-on patch plasty of the left end of MLHV stump (type 3) (Fig. 3). Simulative analysis regarding efficacy of MHV outflow drainage was performed based on the principles of hemodynamics using computational fluid dynamics software (ADINA fluid-structure interaction model, ADINA R\&D, Inc., MA).

\section{Clinical application (Part 3)}

The refined techniques based on the Part 2 analysis were applied to five cases of adult LDLT using MHV reconstruction using a Hemashield graft during a 4-month period from July 2019 to October 2019. The morphological configurational changes at the conduit anastomotic site were analyzed through serial dynamic CT follow-up. 
The surgical techniques to anastomose a Hemashield graft to the right liver graft were as follows: A Hemashield graft with an internal diameter of $10 \mathrm{~mm}$ was used. A small niche was created to enlarge the orifices of MHV tributary stumps, after which an intervening vessel homograft patch was attached for end-to-side anastomosis of MHV branches. The 5-0 Prolene sutures was for vascular anastomosis. Details of the procedures were presented previously. ${ }^{6}$

\section{RESULTS}

\section{Profiles of MHV anastomotic stenosis (Part 1)}

The mean age of 50 recipients was $54.1 \pm 6.1$ years, and male patients were $38(76 \%)$; the number of hepatitis B virus infection-associated patients was 26 (52\%); model for end-stage liver disease score was $16.1 \pm 8.5$; ABO bloodincompatible LDLT was 7 (14\%); and graft-recipient weight ratio was $1.13 \pm 0.21$. All patients are still alive for more than 1 year to date.

The types of conduit-MLHV stump reconstruction technique were type 1 in $23(46.0 \%)$, type 2 in $25(50.0 \%)$, and type 3 in 2 (4.0\%). Noticeable morphological distortion at the conduit-MLHV stump reconstruction on 1-week CT scan was identified in 7 (30.4\%) in type 1, 6 (24.0\%) in type 2 , and none ( $0 \%$ ) in type 3 (Fig. 2). None of them underwent percutaneous stenting to solve the morphological distortion at the conduit-MLHV stump anastomotic site.

\section{Results of simulative analysis (Part 2)}

Type 1 technique could reduce the cross-sectional area at the right end of conduit-MLHV stump anastomosis by $20-40 \%$ depending on the size of MLHV stump. If the transverse diameter of the ovoid MLHV stump was $2 \mathrm{~cm}$, the anastomosis cross-sectional area was reduced by at least $20 \%$ of the $10 \mathrm{~mm}$-sized Hemashield conduit.

Type 2 and type 3 techniques could reduce the crosssectional area at the right end of conduit-MLHV stump anastomosis by $10-30 \%$ depending on the size of MLHV stump. Thus, these two techniques had comparable efficacy on conduit-MLHV stump anastomosis. The size of the MLHV stump turned to be most important factor to achieve secure conduit-MLHV stump anastomosis, which is dependent only on the hepatic vein anatomy of the recipients. In practice, technical widening of the MLHV stump is usually not permissible because of high operative risk.

According to a detailed image analysis of the conduitMLHV stump anastomosis, the most critical point of anastomotic stenosis was the shape of the right end of anastomosis. If it was narrow-angled, it appeared to be vulnerable to making anastomotic stenosis. To make the shape of the right end of anastomosis round, the suture line should be started bidirectionally from the 9 o'clock direction. To apply this suture technique, type 3 technique appeared to be more suitable than type 2 technique.

\section{Results of clinical application (Part 3)}

The technical knacks for type 3 reconstruction learned from Part 2 study can be summarized as follows: cut the conduit end in a dumb-bell shape; attach a $2 \mathrm{~cm}$-long vessel homograft to the left end of MLHV stump; suture bidirectionally from the 9 o'clock direction (Fig. 4).

These knacks were prospectively applied to 5 patients. Follow-up CT scan up to 3 months revealed that noticeable anastomotic stenosis did not develop at all, even in the two patients who had a small-sized MLHV stump (Fig. 5). In all 5 patients, the sources of vessel homograft patch were small cryopreserved iliac artery segments, which were residues after performing bench work reconstruction for MHV tributaries.

\section{DISCUSSION}

Reconstruction of MHV resulted in new demands for prosthetic vascular grafts in the field of LDLT using a modified right liver graft. Regarding availability, prosthetic vascular grafts have a definite advantage of unlimited supply. ${ }^{1-6}$ We previously presented the merit of
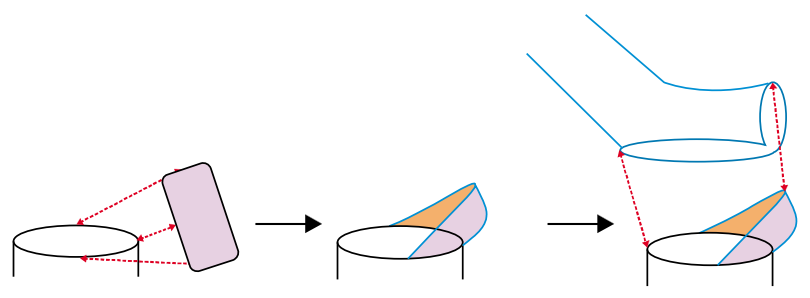

Fig. 4. Schematic illustration of the refined reconstruction techniques of type 3. A rectangular patch indicates a vein homograft. 

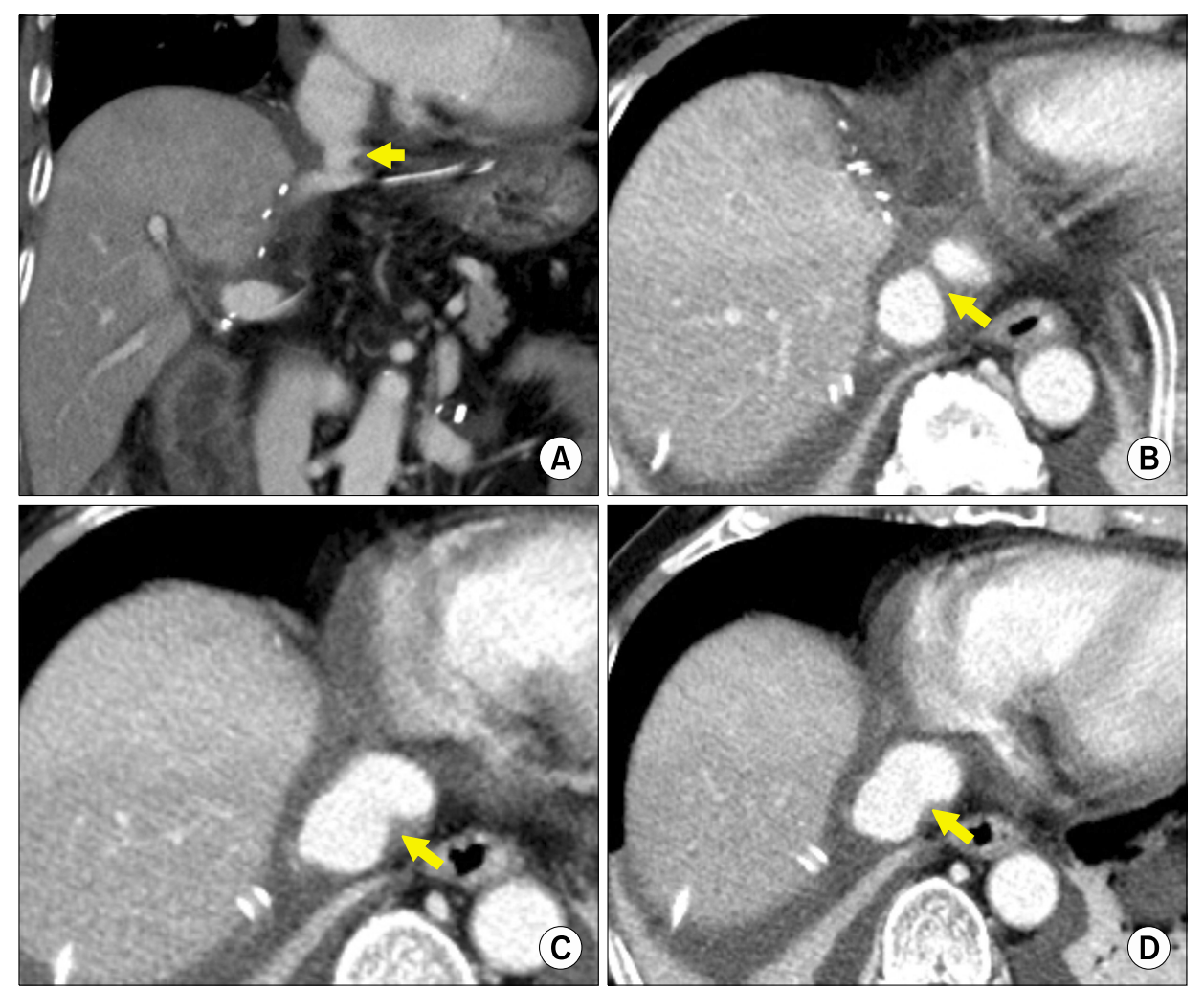

Fig. 5. Dynamic computed tomography images taken 1 week after liver transplantation after application of refined type 3 reconstruction technique. (A) A 3-dimensional reconstruction image shows a neck portion at the left-middle hepatic vein trunk stump (arrow). (B-D) Detailed serial cross-sectional images of the luminal transition from a Hemashield conduit to the left-middle hepatic vein trunk stump (arrows).
Hemashield graft regarding its availability and technical feasibility, and the Hemashield grafts was the most preferable prosthetic vascular graft in our institution. ${ }^{6}$

However, the Hemashield graft is relatively thin-walled, thus being vulnerable to collapse if the pleats were partially disrupted. If such a situation is coincidentally matched with a small-sized MLHV stump, it can result in high incidences of morphological distortion. Therefore, it is necessary to make a technical modification to achieve failure-free MHV reconstruction.

The type 1 reconstruction technique is based on surgeons' intuition. If the size of MLHV stump is large enough, there would be low risk of anastomotic stenosis. ${ }^{2,5}$ However, the size of MLHV stump is widely variable, thus creating chances of potential risk of anastomotic distortion. This was the background reason for performing this study. We accumulated experience on type 2 reconstruction technique for a long period, especially using the ringed Goretex graft. ${ }^{2,5}$ Since the ringed Goretex graft has a thick wall and concentric outer rings, it was resistant to collapse during conduit-MLHV stump anastomosis. Such features of ringed Goretex grafts enabled us to perform both type 1 and 2 reconstruction techniques easily. After using Hemashield grafts, type 1 reconstruction technique did not en- sure secure MHV reconstruction, especially in patients with a relatively small MLHV stump.

After applying the newly refined type 3 reconstruction technique, there was no noticeable anastomotic stenosis. Since the number of study cases was too small for statistical analysis, it is difficult to evaluate its efficacy on patency rate. This is a kind of preliminary study on newly refined technique, thus we believe that further experience and technical evolution will contribute to achieve failure-free MHV reconstruction.

This study has limitations of note. First, it was a smallvolume single-center study, which could potentially introduce selection bias. Second, our refined technique for type 3 reconstruction was not fully matured, thus further validation analysis may affect its technical evolution.

In conclusion, our refined technique to perform conduit-MLHV stump anastomosis helped to reduce the risk of anastomotic stenosis for relatively small MLHV stumps. Further experience and technical evolution will contribute to achieve failure-free MHV reconstruction during LDLT operation. 


\section{CONFLICT OF INTEREST}

No potential financial conflicts or other conflicts of interest exist for any of the authors of this article.

\section{ORCID}

I-Ji Jeong: https://orcid.org/0000-0002-9360-1993

Shin Hwang: https://orcid.org/0000-0002-9045-2531

Tae-Yong Ha: https://orcid.org/0000-0001-9932-0212

Gi-Won Song: https://orcid.org/0000-0002-4235-0434

Dong-Hwan Jung: https://orcid.org/0000-0001-5984-023X

Gil-Chun Park: https://orcid.org/0000-0003-1631-3258

Chul-Soo Ahn: https://orcid.org/0000-0002-3844-3646

Deok-Bog Moon: https://orcid.org/0000-0002-8209-3540

Ki-Hun Kim: https://orcid.org/0000-0002-4016-0995

Young-In Yoon: https://orcid.org/0000-0002-9308-0366

Sung-Gyu Lee: https://orcid.org/0000-0001-9161-3491

\section{AUTHOR CONTRIBUTIONS}

Conceptualization: SH. Data curation: IJI, TYH. Formal analysis: GWS, DHJ. Methodology: GCP, CSA, DBM,
KHK, YIY. Project administration: SH, SGL. Visualization: SH. Writing - original draft: SH, IJI. Writing - review \& editing: SH.

\section{REFERENCES}

1. Hwang S, Lee SG, Lee YJ, Sung KB, Park KM, Kim KH, et al. Lessons learned from 1,000 living donor liver transplantations in a single center: how to make living donations safe. Liver Transpl 2006;12:920-927.

2. Hwang S, Lee SG, Ahn CS, Park KM, Kim KH, Moon DB, et al. Cryopreserved iliac artery is indispensable interposition graft material for middle hepatic vein reconstruction of right liver grafts. Liver Transpl 2005;11:644-649.

3. Sugawara Y, Makuuchi M, Akamatsu N, Kishi Y, Niiya T, Kaneko J, et al. Refinement of venous reconstruction using cryopreserved veins in right liver grafts. Liver Transpl 2004;10: 541-547.

4. Hwang S, Lee SG, Song GW, Lee HJ, Park JI, Ryu JH. Use of endarterectomized atherosclerotic artery allograft for hepatic vein reconstruction of living donor right lobe graft. Liver Transpl 2007;13:306-308.

5. Hwang S, Jung DH, Ha TY, Ahn CS, Moon DB, Kim KH, et al. Usability of ringed polytetrafluoroethylene grafts for middle hepatic vein reconstruction during living donor liver transplantation. Liver Transpl 2012;18:955-965.

6. Park GC, Hwang S, Ha TY, Song GW, Jung DH, Ahn CS, et al. Hemashield vascular graft is a preferable prosthetic graft for middle hepatic vein reconstruction in living donor liver transplantation. Ann Transplant 2019;24:639-646. 